\title{
LXIX. An abstract of the characters of Ochsenheimer's genera of the lepidoptera of Europe; with a list of the species of each genus, and reference to one or more of their respective icones
}

\author{
J.G. Children F.R.S. L.E. F.L.S.
}

To cite this article: J.G. Children F.R.S. L.E. F.L.S. (1829) LXIX. An abstract of the characters of Ochsenheimer's genera of the lepidoptera of Europe; with a list of the species of each genus, and reference to one or more of their respective icones, Philosophical Magazine Series 2, 6:36, 451-464, DOI: 10.1080/14786442908675192

To link to this article: http://dx.doi.org/10.1080/14786442908675192

曲 Published online: 14 Jul 2009.

Submit your article to this journal ¿

Џll Article views: 2

Q

View related articles $\lessdot$ 
LXIX. AnAbstract of the Characters of Ochsenheimer's Genera of the Lepidoptera of Europe; with a List of the Species of each Genus, and Reference to one or more of their respective Icones. By J. G. Chuldren, F.R.S. L.\& E. F.L.S. \&c.

[Concluded from page 335.]

\section{Genus 96. AMPHIDASIS, Ochs., Treitsch.}

\section{(Amphidasis, Phigalia, Nyssia, Duponchel.) Amphidasis, Biston, Stephens.}

Antenno bipectinated in the males, simple in the females; the apex sometimes naked.-Wings strong, generally of a whitish-gray colour, with dark, indistinct bands, and coarse dots; females occasionally apterous.-Body short, and pointed in the males; in the females stout, conical: thorax broad, hairy.

Species.

Icon.

1.Amp.Betularia, Linn.* + Hübn.Geom.tab.33.f.173.(fœm.) 2.-Prodromaria, Fab.* + Hübn.Geom. tab.33.f.172.(mas.) 3.-Hirtaria, Linn.* +.......Hübn.Geom. tab.33. f.175.(mas.) 4.-Pilosaria, Hübn. $\$$...Hübn.Geom. tab.34.f.176.(mas.)

* Biston, Stephens.

+ Amphidasis, Duponchel._." Antenne pectinated in the males, simple in the females. Terminal margin of the wings simple or entire.-Thorax broad, woolly.-Wings thick and small in proportion to the body.-Head sunk beneath the thorax.-Abdomen large, corical.-Maxille none, or scarcely discernible. - Females winged.-Larva long, cylindrical, tubercular; head flat, more or less emarginate on the upper part.-Pupa naked, in the earth."-Duponchel, Lep. de France, tom. vii. part. ii. p. 268. Except that the larvæ are decided loopers, the three species included in this genus by M. Duponchel, might be taken for Bombyces, which, in their perfect state, they very much resemble; they differ from them, however, by the antennæ of the females being entirely filiform, whereas in the Bombyces they are always slightly pectinated, or ciliated.

$f$ Amphidasis, Stephens.

of Prigalia, Duponchel.--" Antenna pectinated in the males, ciliated in the females.-Terminal margin of the wings simple.-Thorax broad, woolly. Abdomen slender.-Wings thin, and large in proportion to the body.-Palpi velvety, not projecting beyond the forehead.-Maxille none, or scarcely discernible.-Females apterous.- Larva cylindrical, of equal size through. out, with a few short hairs; head hemispherical; a bifid tubercle on the eleventh segment.-Pupa naked, in the earth."-Duponch. l. c. p. 296. Duponchel has formed this genus on the single species, pilosaria, which differs from his Amphidases and Nyssiæ, by its slender abdomen, and proportionately wider and thinner wings; and also from the former by the female being apterous. 
Species.

Icon.

5.Amp.Alpinaria, Hübn.* Hübn.Geom. tab. 34. f. 178.(mas. tab. 99. f. 513. (foem.)

6.-Hispidaria, Fab.†* ...Hübn. Geom. tab.34. f.177.(mas.)

7.-Pomonaria, Hübn.† ....Hübn.Geom. tab. 34. f.180.(mas.)

8.-Zonaria, Hübn. †.......Hübn.Geom. tab.34. f.179. (mas.) tab. 99. f. 511 . (fom.)

Genus 97. PSODOS, Ochs., Treitsch.

(Psodos, Duponchel. Psycophora, Kirby, Stephens.)

Palpi very hairy, projecting beyond the forehead._-Maxilla long. Ground-colour of the roings and body black, or very dark; the latter slightly hairy, and slender.

Species.

Icon.

1.Pso.Alpinata, Hübn. $\$$ Hübn.Geom.tab.38. f. 197. (mas.) 2.-Torvaria, Hübn. .......Hübn. Geom. tab. 71. f. 366. 367 . 3.-Horridaria, Fab........Hübn.Geom.tab.60.f.312.(fom.)

4.-Venetaria, Hübn........Hübn.Geom. tab.64.f.329. (mas.) 5.-Trepidaria, Hübn. $\oint$... Hübn. Geom. tab.66. f.343.(fœm.)

Genus 98. FIDONIA, Ochs., Treitsch.

(Fidonia, Ligia, Strenia, Halia, Numeraria, Hibernia,

Fidonia, Bupalus, Anisopteryx, Lampetia, Grammatophora, Azinephora, Cheimatobia, Hercyna, Hyria, Stephens.

Bupalus, Speranza, Curtis.

Wings entire, rounded; sprinkled with dark, minute specks, like dust.-Body slender; back narrow.-Larva stout in proportion to its length, with generally bright coloured dorsal and lateral stripes.-Metamorphosis in a thin web, on the ground, or at a small depth below the surface.

* Nrssia, Duponchel.- " Antennex pectinated in the males, simple in the females.- Terminal margin of the wings simple.-Thorax broad, woolly.Wings thick and small in proportion to the body.-Head sunk beneath the thorax.-Abdomen large, conical.-Palpi velvety, not projecting beyond the forehead.-Maxilla wholly, or nearly wanting.- Females apterous.Larva cylindrical, slightly attenuated at each end, sometimes smooth, sometimes with little tubercles, each carrying a single hair; head hemispherical.Pupa naked, in the earth."-Duponch. l. c. p. 283 . The Nyssiæ are distinguished from the Amphidases (which they very much resemble), not only by the females being apterous, but also by the hemispherical head of the larvæ, which live also exclusively on trees; whereas the larvæ of the latter feed, apparently, in preference on herbaceous plants.

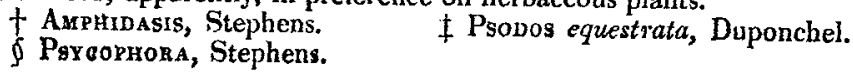


Species.

Icon.

1.Fid.Cebraria, Hübn.......Hübn.Geom. tab.24. f. 129.(mas.)

2.--Hepararia, Hübn.......Hübn. Geom. tab. 11.f. 58.(mas.)

3.-Pinetaria, Hübn.......Hübn.Geom. tab.24. f.130.(fœm.) tab. 100. f. 516. 517. (mas.)

4.- Auroraria, Hübn.*...Hübn. Geom. tab. 12. f. 63.(mas.)

5.-Indigenaria, Treitsch. Hübn.Geom. tab.91.f.168.(fœm.)

6.-Spartiaria, Hübn......Hübn.Geom. tab. 22. f.116.(mas.)

7.-Conspicuaria, Hübn.+ Hübn. Geom. tab. 22. f. 117. 118. (mas.)

8.-Piniaria, Linn.ł........Hübn. Geom. tab. 22. f. 119.120. (mas.) tab. 91. f. 469.470 . (fœem.)

9.-Diversata, Treitsch... Hübn.Geom.tab.39.f. 202.(fœem.) 10.-Jourdanaria, Treitsch. $\S A n$. de la Soc. Linn. de Paris. v. tab. xi. f. $h-n$.

11.-Pennigeraria, Hübn...Hübn.Geom. tab. 70. f.363.(mas.) 12.-Plumistaria, Hüibn.|. .Hübn. Geom. tab. 24. f. 127.(mas.) 13.-Concordaria, Hübn....Hübn.Geom. tab.24. f.126.(mas.) tab. 100. f.518. 519. (fœem.)

14.--Murinaria, Fab.........Hübn.Geom. tab. 21. f.115.(mas.) tab. 25. f. 134. (fœem.)

15.-Atomaria, Linn. .......Hübn.Geom. tab. 25.f.136.(foem.)

16.-Glarearia, Hübn........Hübn.Geom.tab. 25.f.131. (mas.) 17.-Clathrata, Linn. ${ }^{a}{ }^{b}$.......Hübn.Geom. tab.25.f. 132. (fœm.)

* Hyria, Stephens.

† Sreranza, Curtis. - Antennce setaceous, with numerons oblong joints, each joint, in the males, producing two ciliated branches: simple in the females and ciliated beneath.-Maxillee slender, nearly as long as the antennæ.-Labial palpi porrected nearly horizontally, thickly clothed with scales.-Wings, the superior of the male, with a small protuberance on the upper side, near the base.-Head small; abdomen slender.- Legs long.Curtis (Extract).-Type of the genus, Sp. sylvaria, Curtis, Brit. Ent. v. pl. 225. (mas. et foem.)

$\mp$ Bupalus, Stephens.

\$ LigIA, Duponchel.- " Upper wings narrow.-Head surmounted by a tuft of hairs terminating in a point.-Palpi short, obtuse.-Maxille nearly obsolete.-Antenne, in the males, very plumose."-Duponch. Lep. de Fran. tom. vii. part. ii. p. 107.

|| Fidonia, Duponchel._- “All four uings sprinkled with dots more or less minute, forming by their union more or less distinct bands.-Palpi short, often covered with long scales.-Maxillee short, or obsolete.-Antenne very plumose in the males of the principal species."-Duponch. Lep. de France, tom. vii. l. c. supra, p. 107.

If Fidonia, Stephens.

Hercyna, Stephens,

- StrENIA, Duponchel._-“ All four wings marked with longitudinal and transverse lines, or reticulated.-Palpi very short.-Maxille rather long." -Duponch. l. c. supra, p. 112.

18. Fid. 
Species.

Icon.

18.Fid Dilectaria, Hübn.....Hübn. Geom. tab. 8. f. 39.(mas.) 19.-Cararia, Hübn..........Hübn. Geom. tab. 8. f. 38. (foem.) 20.-Immorata, Linn..........Hübn.Geom. tab. 25. f.133.(mas.) 21.-Favillacearia, Hübn.* Hübn.Geom. tab. 26. f.139.(mas.) Curtis, Brit. Ent. i. pl. 33. ot et $q$. 22.-Conspersaria, Fab......Hübn.Geom. tab. 26. f.138.(mas.) 23. - Wavaria, Linn. † ....... Hübn.Geom. tab. 11. f. 55. (fœm.) 24. -Capreolaria, Fab. ......Hübn.Geom. tab. 39.f. 204.(mas.) f. 205. (fœm.)

25.-Plumaria, Hübn. .......Hübn.Geom. tab. 23. f.124.(mas.) 26.-Pulveraria, Linn. \$ I...Hüibn.Geom. tab. 39.f.203.(fœm.) 27.-Aurantiaria, Hübn. ๆ..Hübn.Geom.tab. 35.f.184. (mas.) 23.-Progemmaria, Hübn. IHübn.Geom.tab. 35. f.183.(mas.) 29.-Defoliaria, Linn. I a....Hübn.Geom. tab. 35.f. 182.(mas.) tab. 99. f. 510 . (fœem.)

30.-Aceraria, Hübn.........Hübn.Geom. tab.35. f.185.(mas.) tab. 99. f. 514. (foem.)

31.-Fumidaria, Hübn. .....Hübn. Geom. tab. 101. f. 520. 521. (mas.)

32.-Bajaria, Hübn.........Hübn.Geom. tab. 37. f.194.(mas.) 33.-Leucophrearia, Hübn. ${ }^{b}$ Hübn.Geom. tab. 37.f.195.(mas.) 34.-DAscularia, Hübn. b....Hübn.Geom.tab.36.f.189. (mas.) 35.-Rupicapraria, Hübn. ${ }^{c}$ Hïbn.Geom. tab. 42. f.222.(mas.)

* Bupalus, Stephens. Curtis. - "Antenne setaceous, bipectinated in the males.-Maxillae short, rather broad and flat-LLabial palpi slightly hirsute, shorter than the head, scarcely projecting beyond the eyes.-Wings not angular, nor indented; very much deflexed when at rest.-Body slender."-Curtis. Brit. Ent. i. pl. 33. (Extract.)

+ Grammatophora, Stephens.

f Halua, Duponchel. - "All four wings pulverulent; the superior marked on the anterior margin with three or four spots, fiom each of which springs an indistinct line.-Palpi scarcely projecting beyond the forehead. -Maxille long."-Duponch. l.c. supra, p. 107.

$\oint$ Azinephora, Stephens.

1 Numbra, Duponchel._- All four wings pulverulent, with a transverse band on the middle of the upper.-- Palpi acuminated, and somewhat projecting beyond the forehead.-Maxilla short."-Duponch. l. $c$. supra, p. 107.

I Lampetra, Stephens.

"Hrbernia, Duponchel.- Upper wings more coloured than the lower. - Palpi very short, not projecting as far forward as the forehead. - Maxille none or obsolete. - Legs very long.- Females apterons, or with only the rudiments of wings."-Duponch. l. c. supra, p. 106.

Anisopteryx, Stephens. "Chematobia, Stephens. 
Genus 99. CHESIAS, Ochs., Treitsch.

(Chestas, Duponchel.

Chesias, Pachycnemia, Stephens. Lobophora, Stephens, Curtis.)

Upper wings elliptical or lanceolate; lower oval.-Palpi long, depressed.-Maxilla long.

Species.

Icon.

1.Ch.Spartiata, Fab.*......Hübn.Geom. tab.36. f.187.(mas.)

2.-Polycommata, Hüibn.+ Hübn.Geom. tab.36.f.190.(fœm.) Curtis. Brit. Ent. ii. pl. 81.

3.-Variata, Hübn..........Hübn.Geom. tab. 57.f. 293.(mas.) tab. 73. f. 380 . (foem.) var.

4.--Juniperata, Linn. ......Hübn.Geom. tab. 57. f.294.(mas.)

5.-Obeliscata, Hübn.......Hübn. Geom. tab.57. f.296.(mas.)

6.-Obliquata, Hübn........Hübn.Geom.tab.43. f.225.(foem.) tab. 82. f. 423. (mas.)

7.-Hippocastanata, Hüb. $\neq$ Hübn.Geom.tab. 36.f.186.(mas.)

Genus 100. CABERA, Ochs., Treitsch.

(Cabera, Ephyra, Duponchel.

Cabera, Cyclophora, Stephens.)

All the roings pulverulent, or spotted with multitudes of minute dots, and traversed by from two to four bands. Palpi scarcely projecting beyond the forehead.-Maxilla long.

Species.

Icon.

1.Cab.Pusaria, Linn.\$......Hübn. Geom. tab.17.f.87. (fom.) 2.-Exanthemaria, Esper.§Hübn. Geom. tab. 17.f. 88. (mas. tab. 98. f. 506. (fœm.)

3.-Strigillaria, Hübn.\|...Hübn.Geom. tab.23. f.125.(fœm.) 4.--Onoraria, Hübn. .......Hübn. Geom. tab. 18. f.93.(fœm.) 5.-Punctaria, Linn. I ......Esper.Schm. v. th. tab. vi. f. 5-7. tab. vii. f. 1.2 .

6.-Poraria, Treitsch. ....Hü̈bn. Geom. tab. 13. f.67. (mas.) 7.-Omicronaria, Hübn.ף..Hübn.Geom. tab. 13. f. 65.(mas.) 8.-Ocellaria, Hübn. I ......Hübn. Geom. tab. 13. f.64. (mas.)

* Chesias, Duponchel, Stephens.

+ Loropkora, Stephens, Curtis.-"Antennce rather short, setaceous.Maxillae not very long.-Labial palpi short, distant, incurved, thickly covered with scales.-Wings entire, extended horizontally when at rest; $u p$ per long, somewhat lanceolate; lower small in the males, with a lobe attached at the base of the abdominal margin.-Head small.-Abdomen and legs slender."-Curtis. Brit. Ent. l.c. supra, (Extract.)

I Pachycnemia, Stephens.

If Cabera, Duponchel.

$\S$ CaBElla, Stephens.

I Cyclophora, Stephens.

9. Cab. 
9.Cab.Pendularia, Linn.* †Hübn. Geom. tab. 13. f. 66.(mas.) 10.-Orbicularia, Hübn.*...Hübn. Geom. tab. 12. f.60.(mas.) 11.-Pupillaria, Hübn......Hübn. Geom. tab. 13.f.69.(mas.) 12.-Gyraria, Hübn.........Hübn.Geom. tab.84. f.434.(mas.) 13.-Trilinearia, Bork.* ...Hübn. Geom. tab. 13. f.68.(fœm.)

\section{Genus 101. ACIDALIA, Ochs., Treitsch.}

(Acidalia, Amathia, Larentia, Duponchel. Hemerophila, Ypsipetes, Phibalapteryx, Scotosia, Triphosa, Chemmatobia, Lobophora, Emmelesia, PtYchopoda, Stephens.)

All the wings marked with numerous undulated, transverse parallel lines. - Larva short, stout; generally of a green colour, with pale, longitudinal lines, or reddish streaks: segments of the body, distinct.-Metamorphosis subterranean.

Species.

Icon.

1.Acid.Ochrearia, Hübn...Hübn.Geom. tab.20.f. 110.(mas.) 2.-Rufaria, Hübn..........Hübn.Geom.tab. 21.f.112.(mas.) 3.-Rubricaria, Hübn.. ...Hübn.Geom. tab.21. f.111.(fœem.) tab. 94. f. 487 . (mas.)

4.-Pygmaaria, Hübn.....Hübn.Geom. tab.65.f.335. (mas.) f. 336. (fœm.)

5.-Vittaria, Hübn.........Hübn.Geom. tab.83. f.429.(mas.) 6.-Pusillaria, Hübn.......Hübn.Geom. tab. 19. f.99. (fœem.) 7.-Decolorata, Hübn. f...Hübn.Geom. tab.47.f.243.(fœem.) 8.-Albulata, Hübn. ........Hübn.Geom. tab.50.f.257.(fœm.) 9.-Sylvata, Hübn...........Hübn.Geom. tab.44.f.231.(foem.) 10.-Luteata, Fab.............Hübn.Geom. tab.19.f.103.(fœm.) 11.-Alpestrata, Hübn......Hübn.Geom.tab.62.f.320.(fom.) 12.-Scabraria, Hübn........Hübn.Geom. tab.44.f. 229.(mas.) 13.-Elutata, Hübn. $\oint$.........Hübn.Geom. tab. 43.f.224.(mas.) tab. 74. f. 385. (fœm.)

14.-Impluviata, Hübn.\$ ...Hübn.Geom. tab. 43. f.223.(mas.) 15.-Brumata, Linn. \|........Hübn.Geom. tab.37. f. 191.(mas.) tab. 99. f. 509. (fœem.)

16.-Dilutata, Hübn.........Hübn.Geom. tab. 36. f.188.(mas.) 17.-Lobulata, Hübn. ........Hübn.Geom. tab. 70. f.362.(mas.)

* Cyclorhora, Stephens.

† EPHY RA, Duponchel._- "Base of all the wings pulverulent, with a transverse line, and an omicron, more or less accurately defined, on the centre of the disc, in most of the species.-Palpi slender, very much inclined, and not projecting beyond the forehead.-Maxilla long."-Duponch, Lep. de France, tome vii. part. ii. p. 108.

$\S$ Yrsiperes, Stephens.

$\ddagger$ Emmelesia, Stephens.

if Cheimatobia, Stephens. 
18.Acid.Rupestrata, Fab. ...Hübn.Geom. tab.37.f.192. (mas.) 19.-Candidata, Borkh......Hübn.Geom. tab.19. f.101.(fœem.) 20.-Osseata, Fab.* ..........Hübn.Geom. tab. 19.f.102.(fœm:) 21.-Pallidaria, Hübn......Hübn.Geom. tab. 18. f. 96. (mas.) 22.-Strigaria, Hübn.†......Hübn.Geom. tab. 18. f. 98. (mas.) 23.-Byssinata, Treitsch.

24.-Sericeata, Hübn.........Hübn.Geom. tab. 78. f.404.(mas.) 25.-Hexapterata, Fab.\$....Hübn.Geom. tab. 44. f.232.(mas.) 26.-Sexalata, Bork.\| .....Hübn.Geom. tab.44. f. 228.(mas.) 27.-Rivulata, Hübn. ......Hüibn.Geom. tab.50. f. 259.(mas.) 28.-Blandiata, Hubn. .....Hübn.Geom. tab. 50. f. 258.(mas.) 29.-Rusticata, Fab. .......Hübn.Geom. tab. 46. f.241.(mas.) 30.-Filicata, Hübn.......Hübn.Greom. tab.46. f.238.(fœm.) 31.-Salicaria, Treitsch....Hübn.Geom. tab.53. f.273.(mas.) 32.-Scripturata, Hübn. ....Hübn.Geom. tab.53. f.274.(mas.) 33.-Coraciata, Hübn. ....Hübn.Geom. tab.54. f. 278.(fœem.) 34.-Frustata, Treitsch. 1

35.-Viretata, Hübn. ......Hübn.Geom. tab. 44. f.230.(mas.) 36.-Riguata, Hübn. . . ...Hübn.Geom. tab.69.f.358.(fom.) 37.-Undulata, Hübn. . . . . Hübn.Geom. tab. 51.f.262.(fœm.) tab. 85. f. 4.36. (mas.)

38.-Vetulata, Hübn.**....Hübn.Geom. tab.51.f.263.(mas.) 39. - Fluviata, Hübn. . . . Hübn. Geom. tab.54. f.280.(fœm.) f. 281 . (mas.)

40.-Bilineata, Linn. .. . . .Hübn.Geom. tab.51.f. 264.(fœm.) 41.-Bistrigata, Treitsch.†

42.-Polygrammata, Hubn. Hübn.Geom.tab.54.f. 277. (mas.) 43.-Lignata, Hübn. . . . . Hübn.Greom.tab.52.f.270. (fœem.) 44.-Tersata, Hübn.ł‡. . . Hübn.Geom.tab.52.f. 268. (mas.)

- Ptychopoda, Stephens. tab. 87. f. 448. (fœem.)

+ Acidalia, Duponchel. - "All the wings traversed by parallel lines, sometimes straight, sometimes wavy or sinuated, and varying from three to five, on an uniform ground colour. A point in the middle of each wing, on most species.-Palpi very short.-Maxille long.-Antenne ciliated in the males."-Duponch. Lep. de France, tom. vii. part. ii. p. 108.

f Acid. alis albo flavicantibus, strigis obscurioribus. - Ochs. Treitsch. vi. part. v. p. 36. § LoBOpHoRa, Stephens.

II AмAтHIA, Duponchel._-" Upper wings only traversed by very numerous parallel, wavy lines, separated by bands.-Palpi very shơt.Maxille long.- Lower wings of the males, in many species, with an appendage resembling a third pair of rudimentary wings, near their base, on the inner side."-Duponch. l, c. p. 112.

I Acid. alis anticis fusco virescentibus, fascia obsoleta alba, strigisque obscurioribus; posticis cinereis.-Ochs. Treitsch. l. c. p. 50.

** Scotosia, Stephens.

t+ Acid. alis anticis albido ferrugineis, strigis dentatis fuscis; posticis flavido ferrugineis, linea dentata fusca in medio.-Ochs. Treitsch, vi. part. v. p. 59. \# Phibatanteryx, Stephens.

N.S. Vol. 6. No. 36. Dec. 1829. $3 \mathrm{~N}$ 


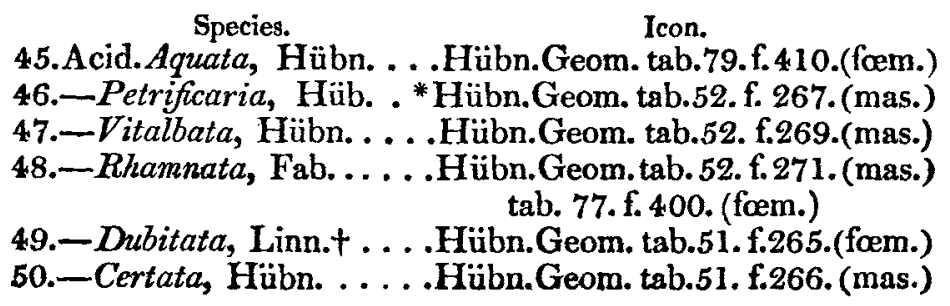

\title{
Genus 102. LARENTIA, Ochs., Treitsch.
}

\author{
(Euboxia, Anaitis, Duponchel. \\ Larentia, Aplocera, Eupithecia, Stephens. \\ Euprthecia, Curtis.)
}

Anterior wings, like those of the preceding genus, with wavy, transverse lines, and frequently a dark transverse band near the centre of the disc.-Larva short, stout, rugose, usually of a greenish colour, with spots or stripes.-Metamorphosis subterranean.

$$
\text { Species. Icon. }
$$

1.La.Cervinaria, Treitsch. $\ddagger$ Hübn.Geom. tab. 62. f.318.(foem.)

2.-Mensuraria, Treitsch.\$Hübn.Geom. tab. 37. f.193.(mas.)

3.-Badiata, Hübn.......Hübn.Geom. tab.56.f.291.(mas.)

4.-Plagiata, Linn.\|. . . . Hübn.Geom. tab.42.f.220.(fom.)

5.-Cassiata, Treitsch. 7

6.-Sororiata, Hübn. . . . .Hübn. Geom. tab.68.f.355. (mas.)

7.-Bipunctaria, Fab. ....Hibn.Geom. tab.53. f.276. (mas.)

8.-Cessiata, Hübn. . . . . Hübn.Geom. tab.53.f.275. (mas.)

9-Sertata, Hübn. . . . . . Hübn.Geom. tab.95.f.489. (mas.)

10.-Flavicinctata, Hübn....Hüibn.Greom. tab.68.f.354. (fœm.)

11.-Molluginata, Hübn. . .Hübn.Geom. tab.71.f.371.(fœem.)

12.-Psittacata, Fab. . . . Hübn.Geom. tab.43.f.227. (mas.)

13. -Cyanata, Hübn... . .. Hübn.Geom. tab.62.f.319. (mas.)

- Hemerophira, Stephens.

† Triphosa, Stephena, - Larantra, Duponchel._"All the wings traversed by a great number of parallel lines, wavy, angular, or indented, and more distinct on the upper than on the lower.-Palpi long, projecting beyond the forehead.-Maxillee long."-Duponch. Lep. de France, tom. vii. part. ii. p. 111.

+ LARENTIA, Stephens. \$ Euboura, Duponchel.-“ Upper wings with a central transverse band, composed of several parallel lines, more or less undulated.-Palpi long, and pointed.-Maxille long."-Duponch. Lep.de Fran. tom. vii. part. ii. p. 109.

|| Aplocara, Stephens.-Anartrs, Duponchel. - " Upper wings only traversed by a great number of angular, parallel lines, divided into bands of three lines each.-Forehead very prominent, but the palpi nevertheless projecting beyond it.-Maxilla short."-Duponch. l. c. p. 111 .

of Lar, alis antiof griseo glaucescentibus, fasciis duabus interruptis fusco ferrugineis ; posticis griseo albidis,-Ochs. Treitsch. vi, part. ii. p. 85 . 
Ochsenheimer's Genera of the Lepidoptera of Europe. 459 Specie:

Icon.

14.La.Rectangulata, Linn.* Hübn.Geom. tab.45. f.255. fom.) tab. 72. f. 372. (mas.)

15.-Isogrammata, Treitsch. $\dagger$

16.-Cydoniata, Borkh. . . . Rösel,Ins.i.th.3.cl.tab.viii. f. 1-3. 17.-Inturbata, Hübn. . . . .Hübn.Geom. tab.90. f.461.(fœem.) 18._Valerianata, Hübn. . .Hübn.Geom. tab.76. f.395.(mas.) 19.-Residuata, Hübn. . . .Hübn.Geom. tab.91.f.467. (fœm.) 20.-Minutata, Hübn. . . . Hübn.Geom. tab.88. f.4.54.(fœm.) 21.-Austerata, Hübn. . . . .Hübn.Geom. tab.89. f.457.(mas.) 22.-Satyrata, Hübn. . . . .Hübn.Geom. tab.85. f.439. (mas.) 23.-Subnotata, Hübn.. . . Hübn.Geom. tab.89.f.458. (fœm.) 24. - Strobilata, Hübn. . . . Hübn.Greom. tab. 87. f.449.(mas.) 450. (fœm.)

25.-Sobrinata, Hübn. . . . . Hübn.Geom. tab.90. f.465.(mas.) 26.-Subumbrata, Hübn. . . .Hübn.Geom. tab.45. f.233.(fœm.) 27.-Oxydata, Treitsch.

28.-Pimpinellata, Hübn. .Hübn.Geom. tab. 86.f.443.(mas.) 44.4. (fœm.)

29.-Exiguata, Hübn. . . . Hübn.Geom. tab.73.f.379.(fœm.) 30.-Consignata, Hübn. . . .Hübn.Geom. tab.47. f.245.(fœm.) 31.-Pusillata, Fab.. . . . . Hübn.Geom. tab.73.f.378.(fœe.) 32.-Hospitata, Treitsch. . .Hübn.Geom. tab.45. f.236. (mas.) 33.-Linariata, Fab. \$ . . . Hübn.Geom. tab.46. f.242.(mas.) Curtis. Brit. Ent. ii. pl. 64.

34.-Irriguata, Hübn. . . . .Hübn.Geom. tab.77.f.397. (mas.) 35.-Innotata, Hübn.. . . . Hübn.Geom. tab.86.f.44l. (mas.) 44.2. (fœm.)

36.-Centaureata, Fab.......Hübn.Greom. tab.46.f.240.(mas.) tab. 88. f. 452. (fom.)

37.-Succenturiata, Linn. ...Hübn.Geom.tab.89.f. 459.(føm.) 38.-Denticulata, Treitsch. II

39.-Sparsata, Hubn. ......Hübn.Geom.tab.77.f. 398.(fom.) 40.-Pygmcata, Hubn........Hübn.Geom.tab.45. f. 234.(fœm.) 4.1.-Nanata, Hubn..........Hübn.Geom.tab. 75. f. 387.(mas.)

- Eupithecia, Stephens.

+ Lar. alis cinereo fuscis, lineis undato albidis._-Ochs. Treitsch.l.c. p. 100.

$\mp$ Lar, alis anticis fuscis, area ferruginea, puncto medio nigro, strigis obsoletis albidis; posticis cinereis, strigis interruptis albidis.-Ochs. Treitsch. l. c. p. 114.

$\$$ Euprtracra, Curtis.-_ Antenne alike in both sexes, rather long, setaceous.-Maxilhe as long as the antenna, slendes. - Palpi projecting obliquely, like a beak, beyond the head, thickly covered with long and broad scales.-Wings entire, horizontal when at rest, superior long, somewhat lanceolate.-Abdomen short, slender.-Lege rather slender."-(Extract.) Curtis l. c. supra.

If Lar. alis albis, limbo strigisque obsoletis fuscescentibus, puncto medio nigro.-Ocks. Treitsch. vi. part i. p. 132 . 
Species.

42.La. Caliginata, Treitsch.*

Icon.

43.-Venosata, Fab. .........Hübn.Geom.tab.47.f. 244.(fom.)

\section{Genus 103. CIDARIA, Ochs., Treitsch.}

(Cidaria, Metantppe, Duponchel.

Crdaria, Harpalice, Electra, Emmelesia, Stephens.)

Wings superior with a dark coloured, transverse band, across the centre of the disc, with its external margin angular.Larva short, thick, each segment with angular spots, the angle pointing towards the head.--Metamorphosis in a slight web, amongst leaves on the ground, or beneath the surface.

$$
\text { Species. }
$$

Icon.

1.Ci.Propugnaria, Treitsch.Hübn.Geom. tab.55. f.286.(foem.) 2.-Aptata, Hübn. ..........Hübn.Geom. tab.67. f.349.(fœe.)

3.-Minorata, Treitsch. +

4.-Graphata, Treitsch. $\ddagger$

5.-Quadrifasciaria,Linn.§Hübn.Geom. tah.55. f.284.(fœm.)

6.-Ferrugaria, Wien.Verz.Hübn.Geom. tab.55.f.258. tab. 89. f. 460. (foem.) (mas.)

7.-Ligistraria, Hübn. ..Hübn.Geom.tab,55. f.282.(foem )

8.--Ocellata, Linn...........Hübn.Geom.táb.48. f.252.(fœm.)

9.-Galiata, Hübn. ......Hübn.Geom. tab.53. f.272.(mas.)

10._Olivaria, Treitsch. ...Hübn.Geom. tab.59. f.307.(føem.)

11.-Miaria, Bork. .........Hübn.Geom. tab.57.f.292.(fœm.)

12.-Tophaceata, Hübn. ...Hüibn.Geom. tab.60. f.309.(mas.)

13. - AEquata, Hübn. ......Hübn Geom. tab.68. f.353.(mas.)

14. - Nebulata, Treitsch.

15.-Populata, Linn. . ......Hübn.Geom. tab.58. f.300. (mas.)

16.-Chenopodiata, Linn....Hüibn.Geom. tab.58. f.299. (mas.)

17.-Achatinata, Hübn. ...Hübn.Geom. täb.58. f.301.(mas.)

18.-Marmorata, Hübn. ...Hübn.Geom, tab.5A. f.279.(fœen.)

19.-Meniaria, Fab. ......Hübn.Geom. tab.5s.f.298.(fœm.)

20.-Fulvata, Hübn. ${ }^{a}$......Hübn.Geom. tab.57.f.297.(mas.)

* Lar. alis plumbeis, atomis, strigisque fuscis.-Ochs. Treitsch. $l$. c. p. 137.

+ Cid. alis albido griseis; anticis fasciis fuscis, albo marginatis, linea externa denticulata alba, puncto medio nigro.-Oehs. Treitsch. vi, part. ii. p. 143.

$\mp$ Cid. alis cretaceis, atomis strigisque numerosis angulatis fuscis, puncto medio nigro.-Ochs. Treitsch. l. c. p. 144.

$\S$ Ctoafta, Stephens.

П Cid. alis cinereo albidis, atomis nigris, fascia media obsoleta,-Ochs. Treitsch:t. c. p. 164.

II Electra, Stephens.

"Croaria, Duponchel.-" Upper Wings traversed across the middle of the disc by a more or less wide band, always bent into one or more salient angles on the outer side.-Palpi projecting beyond the forehead.-Maxilla long." -Duponch. Lep. de France, vii. part. ii. p. 111. 
Ochsenheimer's Genera of the Lepidoptera of Europe. 461

Species.

Icon.

21.Ci.Pyropata, Hübn. .....Hübn.Geom. tab.63. f.328.(fœm.) 22.-Sagittata, Fab. .......Hübn.Geom. tab.60. f.310.(fœm.) 23.-Pyraliata, Fab. .......Hübn.Geom. tab.58. f.302. (mas.) 24.-Derivata, Hübn. ....Hubn.Geom. tab.56. f.289.(fœm.) 25.-Berberata, Fab. . ....Hübn.Geom. tab.56. f.287. (mas.) 26. - Rubidata, Fab. .......Hübn.Geom. tab.56. f.290.(mas.) 27.-Russata, Hübn. . ...... Hübn.Geom. tab.59. f.305.(fœem.) 28.-Suffumata, Hübn. .....Hübn.Geom. tab.59. f.306. (mas.) 29.-Picata, Hübn. .......Hübn.Geom. tab.84. f.435.(fœm.) 30.-Prunata, Linn. .......Hübn.Geom. tab.59. f.304. (mas.) 31.-Silaceata, Hübn. ....Hübn.Geom. tab. 59. f.303.(mas.) tab. 93. f.477. 478. (fœem.)

32.-Reticulata, Fab. ....Hübn.Geom. tab.60. f.308.(fœm.) 33.-Ruptata, Hübn. ....Hübn.Geom. tab.57. f.295.(foem.) 34.-Montanaria, Treitsch.Hübn.Geom. tab.48. f.248.(fœm.) 35.-Alchemillata, Linn.* ..Hübn.Geom.tab.50. f.261 (fœem.) 36.-Hastata, Linn.† ....Hübn.Geom.tab.49. f.256.(foem.) 37.-Tristata, Linn. .......Hübn.Geom. tab.49. f.254.(mas.) tab. 50.f. 260. (fœm.)

38.-Rivata, Hübn. .......Hübn.Geom. tab.79.f.409.(fom.) 39.-Luctuata, Hübn. ....Hübn.Geom. tab.49. f.253.(mas.) 40.-Turbaria, Hübn. ....Hübn.Geom. tab.49. f.255.(foem.)

\section{Genus 104. ZERENE, Ochs., Treitsch.}

(Meranthia, Venilia, Zerene, Corycia, Duponchel. Xerene, Crdaria, Hercyna, Abraxas, Bapta, Stephens.)

Wings superior, with the ground colour nearly white, or yellow, and a more or less interrupted, dark, transverse band. - Larva, thick in proportion to their length; back and sides marked with dots and lines ; motion sluggish.-Metamorphosis in a slight web amongst leaves, or subterranean.

$$
\text { Species. Icon. }
$$

1.Zer.Procellata, Fab. $\neq$..Hübn.Geom. tab.48.f.251.(fœem.) 2.-Fluctuata, Linn.\| ....Hübn.Geom. tab.48. f.249.(mas.) 3.-Stragulata, Hübn. ...Hübn.Geom. tab.65.f.337.(fœm.) 4.-Rubiginata, Fab. ....Hübn.Geom. tab.48.f.250.(føem.)

* Emmerasia, Stephens.

+ MEranippe, Duponchel._" All the wings terminated by a more or less interrupted band. Last joint of the palpi very pointed, scarcely projecting beyond the forehead.-Maxille long."-Duponch. Lep. de France, vii. part. ii. p. 111. $\mp$ Xenene, Stephens.

6 Mrean raia, Duponchel.- " Head, thorax, and base of the upper wings of a deeper colour than the rest.-Palpi very short.-Marille long." Duponch. Lep. de France, vii. part. ii. p.111.

II Cidaria, Stephens. 
Species.

Icon.

5.Zer.Adustata, Fab. ....Hübn.Geom. tab.15. f. 75. (mas.)

6.-Suniata, Hübn.........Hübn.Greom. tab.56. f.288.(mas.)

7.-Albicillata, Linn. .....Hübn.Greom. tab.15. f. 76.(fœem.)

8.-Marginata, Linn. .....Hübn.Geom. tab. 15.f. 80.(mas.)

9.-Maculata, Fab." † ....Hübn.Geom. tab.25.f.155.(mas.)

10.-Melanaria, Linn. .....Hübn.Geom. tab.16. f. 86. (mas.)

11.-Grossulariata, Linn. $\$$ Hübn.Geom. tab. 16. f.81. (foem.)

12.-Ulmaria, Treitsch. . ...Hübn.Geom.tab. 16. f.85. (fom.) tab. 76. f. 391. (mas.) f. 392. (fœrm.)

13.-Pantaria, Linn. ....Hübn.Geom. tab. 16. f. 84.(fcem.) 14.-Cribrata, .Treitsch. ...Hübn.Geom. tab. 16. f. 83. (mas.) 15.-Taminata, Wien.Verz. Hübn.Geom. tab. 17. f. 90.(fœm.) 16. - Temerata, Wien.Verz.\|THübn.Geom. tab.17.f. 91.(mas.) tab. 73. f. 376. (mas.) f. 377 . (fœm.)

Genus 105. MINOA, Ochs., Treitsch.

(Minoa, Cleogene, Tanagra, Duponchel. Minod, Stephens.)

Wings, both on the upper and under surfaces, of one colour.Larva with the body tapering anteriorly, naked, and generally of lively colours; head small--Metamorphosis in a slight web. Divided into two families.

FaM. A.-With rounded wings.

Fam. $B_{\imath}-$ With the anterior wings lanceolate, with faint traces, occasionally, of one or two transverse bands.

Fark. A. Speciés.

Icon.

1. Min. Euphorbiata, Fab. ${ }^{2}$ Hübn. Geom.tab. 15. f. 78. (mas.)

- Hercyna, Stephens.

$\uparrow$ Venilis, Duponchel. - "All the wings sprinkled with little irregular spots, both on the upper and under sides, on a light ground-colour. - Palpi long and velvety. - Maxille long."-Duponch. l. c. p. 110.

f Arraxas, Stephens.

Z ZERENE, Duponchel._- All the wings traversed across the middle by two rows of crowded spots, many of which form larger spots by their union. - Abdomen punctuated.-Palpi very short.-Maxille long, convolute only at the extremity."-Duponch. l.c. p. 110.

II Barta, Stephens.

I Concyria, Duponchel.- " Independent of the rest of the markings, which vary with the species, each wing has a distinct spot in or near the centre of its disc.-Palpi very short.-Maxilla very long."-Duponch. l.c. p. 110.

- Minoa, Duponchel._- All the wings of one colour, both on the upper and under sides; the second wings very much rounded.-Palpishort. - Maxillee long."-Duponch. l. c. p. 112. 
Ochsenheimer's Genera of the Lepidoptera of Europe. $\quad 463$

Species.

Icon.

2.Min.Lutearia, Fab.*. . . Hübn. Geom. tab. 23.f.121.(mas.)

3.-Cherophyllata, Linn. $\dagger_{\dagger}+$ Hübn. Geom.tab. 38.f.196.(mas.) Fam. B.

4.-Griseata, Wien.Verz...Hübn. Geom. tab.41.f. 216.(mas.)

5.-Niveata, Treitsch. . . .Hübn. Geom. tab.41.f.217.(fœm.)

6.-Illibaria, Hübn. . . . Hübn.Geom. tab. 40.f.207. (mas.)

\section{Genus 106. IDEA, Ochs., Treitsch.}

\section{(Siona, Pelronia, Dosithea, Duponchel. IDeA, PtYchopoda, Stephenis.)}

Obs. M. Duponchel, whose profound knowledge of the subject entitles his remarks to more than common attention and respect, says of this genus;- " prejudiced in favour of his (Treitschke's) arrangement of the Phalænidæ, I had intended to adopt it, unaltered, in this work; but on applying it to my own collection, I found that the Author comprehends a host of species, in his genera, which do not possess the characters assigned respectively to them; and that his nineteenth and last genus, which he calls Idcea, is composed of species the most incongruous, such as dealbata, calabraria, ornataria, \&c. : so that one might imagine that he has here brought together all those species for which he could not find a place in either of his preceding eighteen genera, without troubling himself to consider whether or not any analogy exists between them. However, with the exception of this genus, which ought to be abolished, the others appear to rest on solid bases; and I have consequently adopted them, but with the restriction, of referring to each, those species only which really belong to it."-As to the name Idcea, Duponchel very justly observes that it cannot stand, having already been employed to denote an exotic genus of the Papilionidæ.

All the reings with two or three dusky, somewhat arched, and undulated transverse bands, with, generally, between them a point or crescent-shaped spot.-Larva very thin in proportion to their length, almost filiform.-Metamorphosis subterranean.

- Cleogrne, Duponchel._- All the wings of one colour, sometimes very light, sometimes very dark.-Palpi short, velvety.-Maxilla very long."Duponch. l.c. p. 109. + Minoa, Stephens.

$f$ Tavagra, Duponchel. " Superior angle of the first wings, rounded.Body long and thin.-Palpi short.-Maxilla long."-Duponch. l.c. p. 112. 
46 1 Ochsenheimer's Genera of the Lepidoptera of Europe.
Species.
Icon.
1.Id. Dealbata, Linn.* ...Hübn.Geom.tab.41.f.214.(foem.)
2.-Decussata, Wien.Verz..Hübn.Geom.tab. 41.f.213.(mas.)
f. 215 . (fœm.)
3.-Calabraria, Hübn. ...Hübn. Geom. tab. 10. f.49. (fœm.)
4.-Wibicaria, Linn. † .......Hübn. Geom. tab. 10. f. 50. (mas.)
5.-Vincularia, Hübn. ....Hübn. Geom.tab. 78. f.402.(mas.)
6.-Aureolaria, Fab. .... Hübn. Geom. tab. 12. f. 62. (mas.)
7.-Degenerata, Treitsch. Hübn. Geom. tab.11.f. 57. (mas.)
8.-Aversata, Linn. ...... Hübn. Geom. tab. 11. f. 56.(mas.) tab. 75. f. 389. (fœm.)

9.-Suffusata, Treitsch. $\neq$

10. -Remutata, Linn. . . . Hübn. Geom.tab. 18. f. 98. (fœm.) 11.-Mutata, Treitsch... . . Rösel, I. th. 3. cl. tab. 11.f. 1-3. 12.-Submutata, Treitsch. $\$$

13.-Immutata, Linn. . . . Hübn. Geom.tab. 20.f.108.(mas.)

14. -Incanata, Linn. . . . . Hübn. Geom. tab. 19.f.100.(mas.) tab. 20. f. 106. (fœem.)

15.-Ornata, Fab.\| . . . . . Hübn. Geom. tab. 14. f. 70. (mas.) 16. -Decorata, Wien. Verz.qHübn. Geom. tab. 14. f. 71. (mas.) 17.- Reversata, Treitsch.a

18.-Bisetata, Borkh. . . . .Hübn. Geom. tab. 14. f. 73.(foem.) 19.-Scutulata, Borkh. .....Hübn. Geom.tab. 14.f. 72. (fœm.) 20.-Moniliata, Fab. . . . . Hübn. Geom. tab.12. f. 59. (fœem.) 21.-Lavigata, Fab. . . . . Hübn. Geom. tab.14.f. 74. (foem.)

At length we have completed our extracts from the Schmetterlinge Von Europa, as far as we have yet received the work. When the third part of the sixth volume shall reach us, we propose to resume our labours, in continuation. Till when, we heartily bid our entomological readers farewell.

* IDEA, Stephens.-Scoria, Duponchel._- “Nervures of the wings, very strong.-Abdomen long, linear.-Palpi with the last joint very acute, projecting beyond the forehead.-Maxilla very long."-Duponch. Lep. de Fran. tom. vii. part. 2. p. 112.

+ Pellonia, Duponchel._- All the wings traversed by a narrow band towards the centre of the disc, - the band often separating into two lines. -Antenne and legs very long.-Palpi obtuse, not projecting beyond the forehead.-Maxilla long."-Duponch. l. c. p. 109.

† Id. alis virescenti flavidis, lineis obsoletis fuscescentibus, puncto medio nigro.-Ochs. Treitsch. vi. part. 2. p. 272.

$\$$ Id. alis albidis, atomis cærulescentibus; anticis maculis costæ lineisque obsoletis fuscis.-Ochs. Treitsch.l.c. p. 277 .

II Dositura, Duponchel.- "All the wings with a point in the centre, on an uniform ground, and traversed near the extremity by a sinuous line, usually accompanied by confluent spots.-Palpi very short.-Maxilla long. - Antenne in the males rather ciliated than pectinated."-Duponch.l.c. p. 108.

$T$ PXXchOPODA, Stephens.

a Id. alis pallide flavis, margine externo fusco, lineaque undata albida, puncto medio nigro.-Ochs. Treitsch.l.c.p. 286. 